\title{
Ipsilateral femoral neck and shaft fractures: a retrospective analysis of two treatment methods
}

\author{
Roop Singh • Rajesh Rohilla - Narender Kumar Magu • \\ Ramchander Siwach • Virender Kadian • \\ Sukhbir Singh Sangwan
}

Received: 13 December 2007/Accepted: 8 July 2008/Published online: 7 August 2008

(C) Springer-Verlag 2008

\begin{abstract}
Background No consensus exists regarding the optimal treatment of ipsilateral femoral neck and shaft fractures. The three major issues related to these fractures are the optimal timing of surgery, which fracture to stabilize first, and the optimal implant to use. In an effort to find answers to these three key issues, we report our experience of managing 27 patients with ipsilateral femoral neck and shaft fractures by using two different treatment methods, i.e., reconstructiontype intramedullary nailing and various plate combinations. Materials and methods We divided patients into two groups. Group I included 15 patients $(13$ males and 2 females) who were operated with cancellous lag screws or dynamic hip screws (DHS) for fractured neck and compression plate fixation for fractured shaft of the femur. Group II included 12 patients (11 males and 1 female) who were operated with reconstruction-type intramedullary nailing.

Results Mean age was 33.2 and 37.9 years in group I and II, respectively. Mean delay in surgery was 5.9 and 5.4 days in group I and II, respectively. Average union time for femoral neck fracture in groups I and II were 15.2 and 17.1 weeks, respectively; and for shaft fracture these times were 20.3 and 22.8 weeks, respectively. There were 13 (86.6\%) good, $1(6.7 \%)$ fair and $1(6.7 \%)$ poor functional results in group I. There were $10(83.3 \%)$ good, $1(8.3 \%)$ fair and 1 $(8.3 \%)$ poor functional results in group II.

Conclusions Both of the treatment methods used in the present study achieved satisfactory functional outcome in
\end{abstract}

R. Singh $(\bowtie) \cdot$ R. Rohilla $\cdot$ N. K. Magu $\cdot$ R. Siwach

V. Kadian - S. S. Sangwan

Department of Orthopaedic Surgery, Paraplegia

and Rehabilitation, Pt. B.D. Sharma PGIMS,

9-J/52, Medical Enclave, Rohtak 124001, Haryana, India

e-mail: drroopsingh@rediffmail.com these complex fractures. Fixation with plate for shaft and screws or DHS for hip is easy from a technical point of view. Choice of the treatment method should be dictated primarily by the type of femoral neck fracture and the surgeon's familiarity with the treatment method chosen. The femoral neck fracture should preferably be stabilized first, and a delay of 5-6 days does not affect the ultimate functional outcome.

Keywords Reconstruction nailing · Plating · Ipsilateral femoral neck and shaft fracture . Dynamic hip screw · Lag screw

\section{Introduction}

Ipsilateral femoral neck and shaft fractures occur in $2.5-9 \%$ of femur fractures $[6,12,14]$. Most are encountered in high-energy trauma [5, 6, 14]. Victims are usually young, with multiple associated injuries [12, 14]. The diagnosis of the neck fracture is delayed in $19-31 \%$ of patients [14]. The treatment of ipsilateral femoral neck and shaft fractures is difficult, and there are many protocols for the management of these fractures. Treatment options include: (1) antegrade femoral nailing of the shaft with cancellous screws placed anterior to the nail for fixation of the neck [1, 14]; (2) reconstruction-type intramedullary nailing [6, 10]; (3) various plate combinations [including a dynamic hip screw (DHS) and long side plate configuration, a hip screw with a short side plate for the neck and separate plate for the shaft, or cancellous screws for femoral neck and a plate for the shaft] [5, 14]; (4) retrograde intramedullary nailing of the shaft and screw fixation of the neck [8]. Each method has its own advantages and disadvantages. No consensus exists regarding the optimal treatment of these complex fractures [5]. The three major 
issues related to these fractures are optimal timing of surgery, which fracture to stabilize first, and the optimal implant to use [6]. This retrospective study reports our experience of managing 27 patients with ipsilateral femoral neck and shaft fractures using two different treatment methods, i.e., reconstruction-type intramedullary nailing and various plate combinations, in order to address these three key issues.

\section{Materials and methods}

Between January 2000 and December 2006, we treated 27 patients with ipsilateral femoral neck and shaft fractures. All patients were injured after high-energy trauma in road traffic accidents. Thirteen patients had injuries to other parts, viz. abdomen, chest, head and other limbs. None of the patients had open or pathological fracture. We divided patients into two groups (Table 1). Group I included 15 patients (13 males and 2 females) who were operated with cancellous lag screws or DHS for fractured neck and compression plate fixation for fractured shaft of the femur. Mean age was 33.2 years (range, 22-45 years; SD, 6.2 years). Thirteen patients had basicervical and two had transcervical femoral neck fractures. Thirteen patients had Garden type II and two had Garden type III fractures. Eleven patients had femoral shaft fracture in the middle third and four had it in the distal third. According to the Hansen-Winquist classification [4], three of the diaphyseal fractures were type I, seven were type II, one was type III, and four were type IV. As per AO classification, three fractures were type $32 \mathrm{~A}$, seven were $32 \mathrm{~B}$, and five fractures were 32C. Group II included 12 patients (11 males and 1 female) who were operated with reconstruction-type intramedullary nailing. Mean age was 37.9 years (range, 22-51 years; SD, 11.6 years). Ten patients had basicervical, one had transcervical, and one had subcapital femoral neck fractures. Ten patients had Garden type II and two had Garden type III fractures. Nine patients had a femoral shaft fracture in the middle third, one in the proximal third and two in the distal third. According to Hansen-Winquist classification, five of the diaphyseal fractures were type I, five were type II; one was type III, and one was a segmental fracture. As per AO classification, eight fractures were type $32 \mathrm{~A}$, three were $32 \mathrm{~B}$, and one was $32 \mathrm{C}$. All patients were initially managed in the Accident and Emergency Department. Life-threatening conditions were evaluated and managed first by general surgeons and neurosurgeons. Vital signs were stabilized. Temporary skeletal traction via a Steinmann pin was used in patients who could not be operated on immediately. We stabilized femoral neck fracture first in patients operated with various plate combinations. A temporary stabilization with guide
Table 1 Comparison of group I and II

\begin{tabular}{|c|c|c|c|}
\hline Criteria & Group I & Group II & $P$-value \\
\hline \multicolumn{4}{|l|}{ Age } \\
\hline Mean & 33.2 & 37.9 & \multirow[t]{2}{*}{ NS (0.18) } \\
\hline SD & 6.2 & 11.6 & \\
\hline \multicolumn{4}{|l|}{ Sex } \\
\hline Males & 13 & 11 & \multirow[t]{2}{*}{ NS $(0.68)$} \\
\hline Females & 2 & 1 & \\
\hline \multicolumn{4}{|l|}{ Femoral neck fracture classification } \\
\hline Garden II & 13 & 10 & \multirow[t]{2}{*}{ NS $(0.80)$} \\
\hline Garden III & 2 & 2 & \\
\hline \multicolumn{4}{|c|}{ Femoral shaft fracture: Hansen-Winquist classification } \\
\hline Type I & 3 & 5 & \multirow[t]{5}{*}{ NS $(0.23)$} \\
\hline Type II & 7 & 5 & \\
\hline Type III & 1 & 1 & \\
\hline Type IV & 4 & - & \\
\hline Segmental & - & 1 & \\
\hline Average operation time (min) & 72.5 & 115.2 & $P<0.0001$ \\
\hline \multicolumn{4}{|l|}{ Femoral neck fracture } \\
\hline Union & 15 & 11 & \multirow[t]{2}{*}{ NS (0.90) } \\
\hline Nonunion & - & 1 & \\
\hline \multicolumn{4}{|l|}{ Femoral shaft fracture } \\
\hline Union & 13 & 9 & \multirow[t]{2}{*}{ NS (0.78) } \\
\hline Delayed union & 2 & 3 & \\
\hline $\begin{array}{l}\text { Femoral neck fracture- } \\
\text { average union time (weeks) }\end{array}$ & 15.26 & 17.08 & NS (0.17) \\
\hline $\begin{array}{l}\text { Femoral shaft fracture- } \\
\text { average union time (weeks) }\end{array}$ & 20.26 & 22.8 & NS $(0.13)$ \\
\hline Osteonecrosis of femoral head & 0 & 1 & NS $(0.90)$ \\
\hline $\begin{array}{l}\text { Femoral neck fracture- } \\
\text { loss of reduction }\end{array}$ & 0 & 1 & NS (0.9) \\
\hline Reoperation rate & 0 & 4 & NS (0.06) \\
\hline \multicolumn{4}{|l|}{ Functional outcome } \\
\hline Good & 13 & 10 & \multirow[t]{3}{*}{ NS (1.0) } \\
\hline Fair & 1 & 1 & \\
\hline Poor & 1 & 1 & \\
\hline
\end{tabular}

$N S$ not significant, $S D$ standard deviation

wires was done in patients with displaced neck fractures in order to prevent further displacement, and this was followed by stabilization of the shaft and definitive fixation of the neck fracture. In reconstruction nailing, we temporarily stabilized the neck fracture with two guide wires; this was followed by the insertion of the nail, proximal locking and distal locking.

All patients received perioperative antibiotic prophylaxis in the form of inj. cefoperazone + sulbactam $1 \mathrm{gm}$ from $1 \mathrm{~h}$ before surgery until the seventh postoperative day. On the second postoperative day, range of movement exercises were started. Touch-toe weight bearing was allowed using a frame or crutches after stitch removal. 
Patients were followed at monthly intervals up to six months, then at three monthly intervals up to one year, and then every six months up to the last follow-up. The followup study included both clinical and radiological evaluations. Progressive weight bearing was allowed after the appearance of callus on radiographs. Union was defined as painless full weight bearing on the affected limb with the presence of radiologic consolidation in both anteroposterior and lateral views. Delayed union was defined as a fracture that was not united after 24 weeks. The functional results of the patients were assessed with the system used by Friedman and Wyman [3]. A good result required no limitation in the activities of daily living (ADL), no pain, and a $<20 \%$ loss of hip or knee motion. A fair result indicated mild limitation of ADL, mild-to-moderate pain, and a $20-50 \%$ loss of motion. A poor result was associated with moderate limitation of ADL, severe pain, and a $>50 \%$ loss of motion.

Ethical and legal procedure

The protocol was approved by an ethics committee and thus meets the standards of the Declaration of Helsinki in its revised version of 1975 and amendments made to it in 1983, 1989 and 1996 (JAMA 1997; 277:925-6).

\section{Results}

\section{Group I}

Operations were performed within a mean of 5.9 days (range, 2-11 days) following trauma on an ordinary operation table under image intensifier control. Various plate combinations were used: DHS long plate in two patients, lag screws and DCS in three, lag screws and low-contact dynamic compression plate in nine, and lag screws and locking compression plate in one patient. All patients were operated using a closed technique for fractured neck and with biological exposure of the fractured shaft of the femur, with the fractured neck femur stabilized first. Further devascularization of fragments and periosteum stripping were carefully avoided. In one patient, nonanatomical reduction of the displaced neck fracture resulted in unacceptable rotational deformity of the limb after subsequent plating of the fractured shaft of the femur. This was detected and corrected during the same surgical sitting. Average operation time (skin incision to skin closure) was $72.5 \mathrm{~min}$ (range, 59-88 min; $\mathrm{SD}, 8.4 \mathrm{~min}$ ). Patients were followed up for a mean of 24.2 months (range, 19-34 months; SD, 3.5 months). All femoral neck fractures united at an average union time of 15.2 weeks (range, 14-17 weeks; SD, 0.9 weeks)
(Fig. 1a-d). Neither osteonecrosis of femoral head nor proximal fracture nonunion was observed. Two patients had delayed union of femoral shaft fractures. Average union time for femoral shaft fractures was 20.3 weeks (range, 18-30 weeks; SD, 3.9 weeks). There were 13 $(86.6 \%)$ good, $1(6.7 \%)$ fair and $1(6.7 \%)$ poor functional results in group I. One patient had poor functional outcome because of a $>50 \%$ loss of motion at the knee joint. No patient had limb length discrepancy.

\section{Group II}

Operations were performed within a mean of 5.4 days (range, 2-10 days) following trauma under image intensifier control. Both femoral neck and shaft fractures were operated using closed techniques in all patients, except for one who had open reduction of the shaft fracture. Average time of operation was 115.2 min (range, 75-139 min; SD, $18.6 \mathrm{~min}$ ). Patients were followed up for a mean of 27.1 months (range, 20-31 months; SD, 3.3 months). We faced technical difficulties during the operations in four patients. Anatomical reduction of the femoral neck fracture could not be achieved in one patient. Proper screw placement in the neck could not be achieved in two patients. The guide wire could not be inserted in the distal fragment with the closed technique in one patient who had open reduction of the femoral shaft fracture. One patient had nonunion with coxa vara of $100^{\circ}$ and was reoperated three months after the initial procedure. Valgus osteotomy with a $120^{\circ}$ blade plate was performed to correct coxa vara and Ender nailing was performed for a uniting fractured femur shaft. However, nonunion persisted and the patient had revision valgus osteotomy with a DHS valgus osteotomy plate. Fractured neck and shaft of femur united 31 and 20 weeks after the initial procedure in this patient. All femoral neck fractures united at an average union time of 17.1 weeks (range, 13-31 weeks; SD, 4.9 weeks). Three patients had delayed union of the femoral shaft fracture, and dynamization was needed in two patients. Average union time for femoral shaft fractures was 22.8 weeks (range, 17-33 weeks; SD, 4.7 weeks) (Fig. 2a-d). Avascular necrosis of the head of the femur developed in one patient at one year (Fig. 3a-c). There were $10(83.3 \%)$ good, 1 (8.3\%) fair and $1(8.3 \%)$ poor functional results in group II. One patient had poor functional outcome because of a $>50 \%$ loss of motion at knee and hip joint. One patient had a limb length discrepancy of $1 \mathrm{~cm}$. A rotational misalignment of $>10^{\circ}$ was not observed in any of the patients.

Statistical analysis

Data were analyzed using the chi-square test with Yates' correction and Student's $t$-test. For all tests, a probability of 
Fig. 1 a Preoperative radiograph of a 31-year-old male showing a Garden type II fractured neck of the femur. b Preoperative radiograph of the same patient showing a Hansen-Winquist type IV femoral shaft fracture. c Followup radiograph at 18 months of the same patient showing the union of the femoral neck fracture after fixation with three lag screws. d Follow-up radiograph at 18 months of the same patient, showing good consolidation of the femoral shaft fracture after osteosynthesis with a bridging locking compression plate
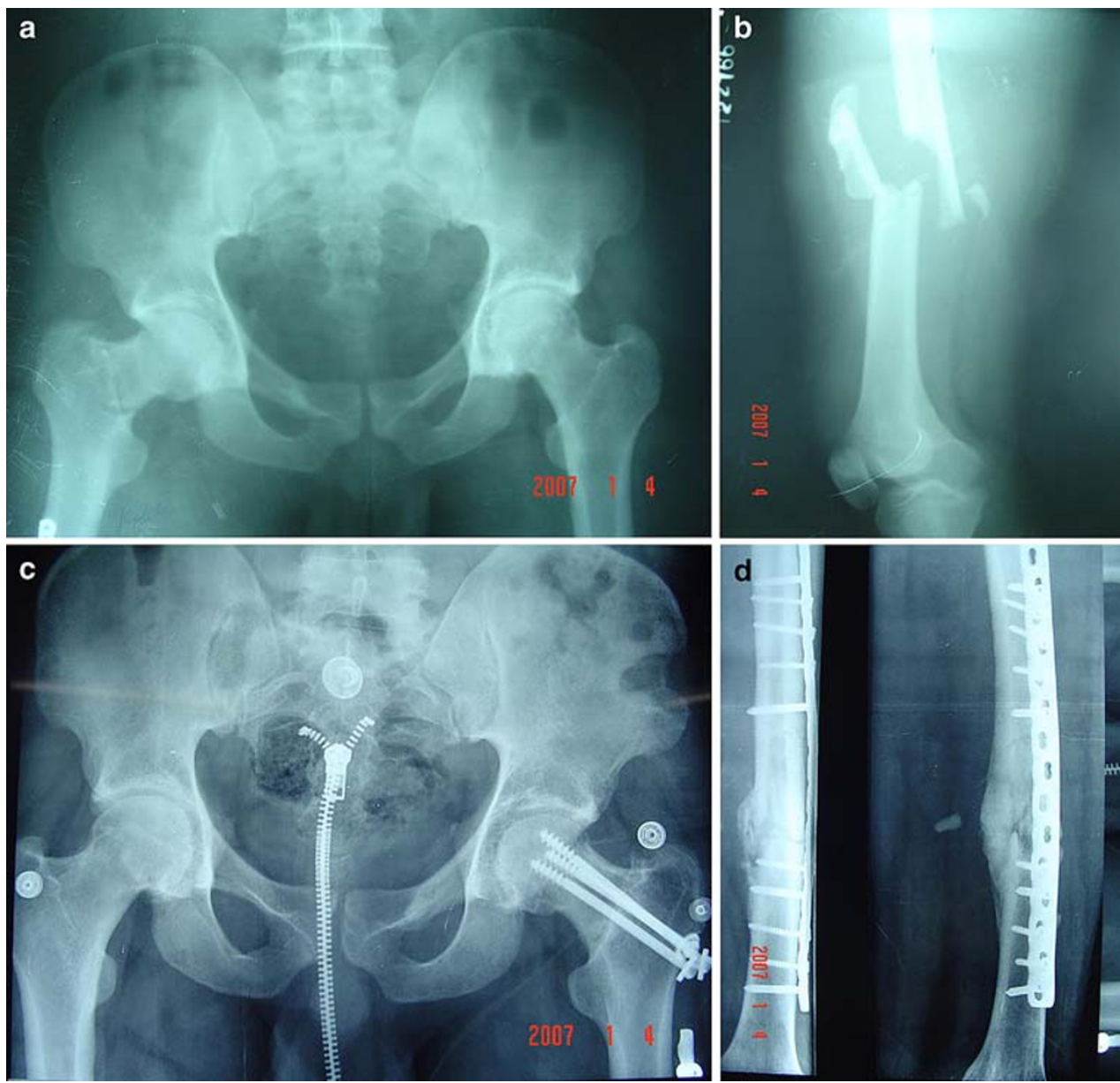

$<0.05$ was considered significant. Chi-square testing showed no significant differences between groups I and II with respect to gender, type of femoral neck fracture, type of femoral shaft fracture, functional outcome, and complications. An unpaired $t$-test revealed no preoperative significant differences between groups I and II with respect to the patient's age (Table 1).

\section{Discussion}

Ipsilateral femoral neck and shaft fractures are rare and challenging. The majority of the patients in the present series were young males with high-energy trauma, as also reported in the literature $[1,5,6]$. Femoral neck fractures were most often basilar in the present series and in other series $[1,6]$. Femoral neck fracture is unrecognized in the initial examinations of $20-30 \%$ of patients [13]. However, we did not observe any missed femoral neck fractures, probably because of our standard protocol of roentgenographic evaluation of the pelvis and both hips in all femoral shaft fractures. Operations were performed within 2-11 days following trauma. The timing of operative fixation was often dictated by the patient's status as a multiple trauma victim in the present series. Delay in treatment was generally because of the associated injuries (head, chest or abdominal) [6]. The rate of avascular necrosis of the femoral head was 4\%, and a delay of 5-6 days in fixation of the neck fracture did not seem to increase this complication rate in the present series. The patient who developed AVN was operated eight days after trauma (Fig. 3a-c). The rate of avascular necrosis of femoral head was lower $(3 \%)$ in ipsilateral neck fractures than in solitary neck fractures $(10 \%)$, and was not associated with the delay in diagnosis or the time of operation in a meta-analysis by Alho [1]. Emergency fixation of the fractured neck of femur in this combined injury pattern, unlike isolated femoral neck fractures, may be unnecessary [16]. Though there is confusion regarding which fracture should be managed first, there appears to be a general consensus regarding the seriousness of the complications involving femoral neck fractures $[5,6,11]$. In a series utilizing a standard protocol of plate fixations for diaphyseal fractures and lag screws or DHS fixations for the hip fractures, Hung et al. reported that the order of fixation of the fractures may not be very important [5]. 
Fig. 2 a Preoperative radiograph showing a Garden type II fractured neck of a femur. b Preoperative radiograph of the same patient, showing a Hansen-Winquist type I femoral shaft fracture and an undisplaced intercondylar fracture of the distal end of the femur. c Femoral neck and shaft fractures were stabilized with reconstruction-type intramedullary nailing. The intercondylar fracture was fixed with a lag screw. A follow-up radiograph at 28 months showed the union of the femoral neck fracture. d Anteroposterior radiograph showing the good consolidation of the shaft and the intercondylar fracture of the femur
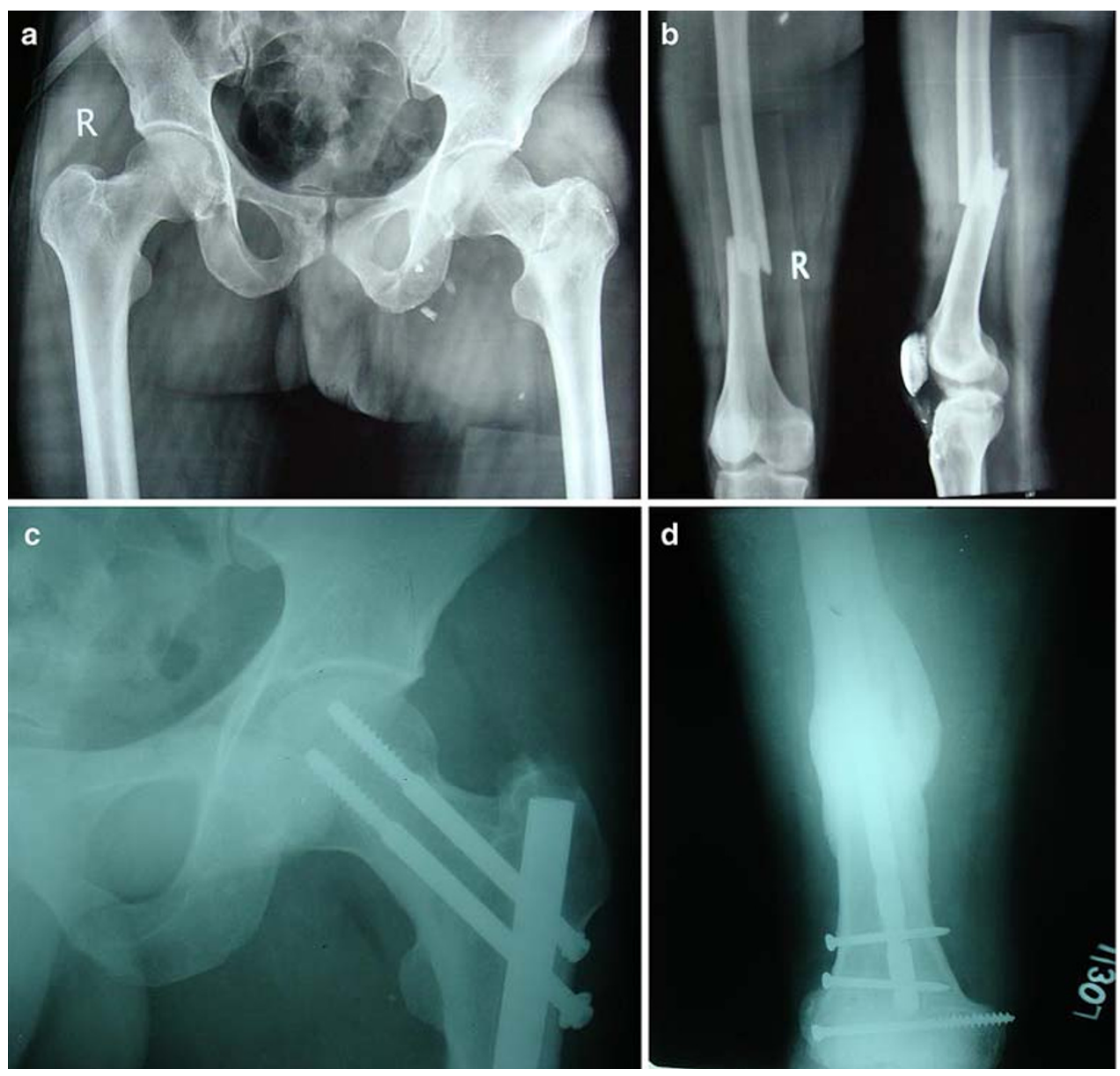

Fig. 3 a Preoperative radiograph showing a Garden type II fractured neck of a femur. b Preoperative radiograph of the same patient showing a Hansen-Winquist type II femoral shaft fracture. c Anteroposterior radiograph showing the union at the neck and shaft fractures. However, union was complicated by stage 3 avascular necrosis of the femoral head
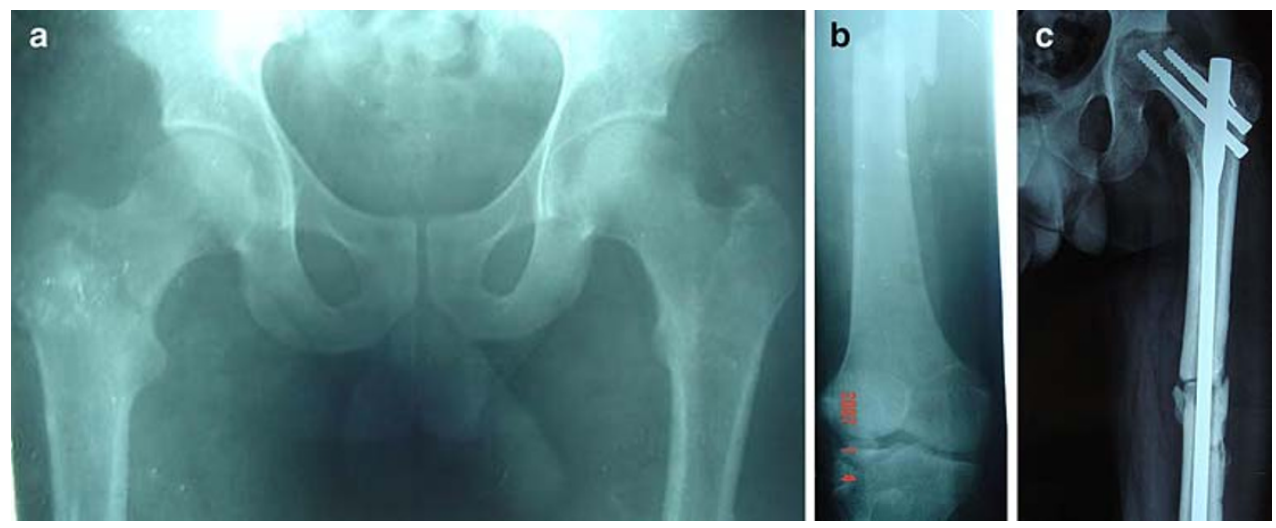

We stabilized femoral neck fractures first in patients operated with various plate combinations. This protocol is satisfactory in patients with undisplaced neck fractures, as further displacement of the neck fracture is prevented. However, in displaced neck fractures, this protocol may result in unsatisfactory reduction of the neck fracture due to inadequate control over the distal fragment at the neck fracture site. Moreover, if the neck fracture is not reduced anatomically, the anatomical reduction at the shaft fracture site during plating may result in unacceptable rotational deformity of the limb. A temporary stabilization with guide wires is recommended in patients with displaced neck fractures in order to prevent further displacement, and this should be followed by stabilization of the shaft and definitive fixation of the neck fracture. Prior stabilization of the shaft aids in reducing the displaced neck fracture. During reconstruction nailing, we temporarily stabilized the neck fracture with two guide wires, and followed this with the insertion of the nail, proximal locking and distal locking. 
There is still no consensus on the optimal treatment method for these complex fractures. In a meta-analysis of the reports published in the literature, the locked intramedullary nails or reconstruction nails yielded results that were superior to those for combinations of plates [1]. The plate series was associated with more frequent infections and nonunions, while the nail fixations were complicated by rotatory malalignments and shortenings [1]. However, the difference between the two treatment methods with respect to union, complications and functional outcome was not significant in the present series. The average time for femoral neck and shaft union in the present series was consistent with that reported in other series $[2,6]$. The choice of the implant in the present series was influenced by the surgeon's preference. We have found reconstruction nailing to be technically demanding. We experienced technical difficulties in four patients undergoing reconstruction nailing. It is difficult to achieve reductions in displaced femoral neck fractures in such complex injuries with reconstruction nailing, and varus nonunion or malunion can occur, as observed in one patient in the present series. The stability of neck fixation may be insufficient [7]. In a retrospective analysis of 13 patients with ipsilateral neck and femoral shaft fractures who had healing complications, Watson and Moed reported that $75 \%$ of the femoral neck nonunions that occurred in these 13 patients developed after the use of reconstruction-type intramedullary nails [12]. The biomechanical advantage of reconstruction nailing is outweighed by the technical difficulties involved in accurately placing the proximal screws into the head and neck [11]. In the present series, we also had similar problems in two patients. We are of the opinion that, from a technical point of view, it is much easier to fix with plate and screws or plate and DHS for ipsilateral neck and shaft fracture than with intramedullary nailing with screws or reconstruction nails. Other authors have also had similar opinions $[2,5]$.

We performed intertrochanteric valgus osteotomy to correct varus setting of the neck fracture in one complicated case of reconstruction nailing. Watson and Moed also reported union after intertrochanteric valgus osteotomy in femoral neck nonunions in patients with ipsilateral neck and femoral shaft fractures who had healing complications [12]. In a series of 16 patients of ununited ipsilateral femoral neck and shaft fractures, the author advocated subtrochanteric valgus osteotomy with sliding compression screws for neck fractures, and dynamic compression plating with supplementary cancellous bone grafting for shaft fracture stabilization [15]. The author considered the surgical procedure to be relatively simple [15].

The goal of any treatment plan should be anatomic reduction of neck fracture and stable fixation of both fractures, so the patient can be mobilized early [6]. Both of the treatment methods used in the present study achieved satisfactory functional outcome in these complex fractures. A reconstruction nail is advantageous in terms of possible closed antegrade nailing with minimal incision, and reduced blood loss and biological fixation of both fractures with a single implant [6,9]. Fixation with plates for the shaft and screws or DHS for the hip is easy from a technical perspective $[2,5]$. The limitations of the present study include the small number of patients in each group and the potential for user bias, because the surgeon could not be blinded with respect to the method used. These limitations notwithstanding, this retrospective study showed that both treatment methods are reliable options in the management of ipsilateral femoral neck and shaft fractures. In our opinion, the choice of the treatment method should be dictated primarily by the type of femoral neck fracture and the surgeon's familiarity with the treatment method chosen. Reconstruction nailing should not be preferred in displaced femoral neck fractures, because of difficulties in reducing the fracture and its maintenance during nail insertion. We are of the opinion that the femoral neck fracture should preferably be stabilized first. A short delay of 5-6 days in stabilizing ipsilateral femoral neck and shaft fractures does not seem to affect the ultimate functional outcome.

Conflict of interest statement No financial grants were received for the study. No other people or organizations were associated with the work or influenced it.

\section{References}

1. Alho A (1996) Concurrent ipsilateral fractures of the hip and femoral shaft: a meta-analysis of 659 cases. Acta Orthop Scand 67:19-28

2. Chen $\mathrm{CH}$, Chen TB, Cheng YM, Chang JK, Lin SY, Hung SH (2000) Ipsilateral fractures of the femoral neck and shaft. Injury 31:719-722

3. Friedman RJ, Wyman ET (1986) Ipsilateral hip and femoral shaft fractures. Clin Orthop Relat Res 208:188-194

4. Hansen ST, Winquist RA (1978) Closed intramedullary nailing of fracture of the femoral shaft. Part II. Technical considerations. In: The American Academy of Orthopaedic Surgeons: Instructional course lectures, vol 27. CV Mosby, St. Louis, MO

5. Hung SH, Hsu CY, Hsu SF, Huang PJ, Cheng YM, Chang JK, Chao D, Chen CH (2004) Surgical treatment for ipsilateral fractures of the hip and femoral shaft. Injury 35:165-169

6. Jain P, Maini L, Mishra P, Upadhyay A, Agarwal A (2004) Cephalomedullary interlocked nail for ipsilateral hip and femoral shaft fractures. Injury 35:1031-1038

7. Kao HK, Wu CC, Lee PC, Su CY, Fan KF, Tseng IC (2006) Ipsilateral femoral neck and shaft fractures treated with RussellTaylor reconstruction intramedullary nails. Chang Gung Med J 29(1):79-85

8. Oh CW, Oh JK, Park BC, Jeon IH, Kyung HS, Kim SY, Park IH, Sohn OJ, Min WK (2006) Retrograde nailing with subsequent screw fixation for ipsilateral femoral shaft and neck fractures. Arch Orthop Trauma Surg 126(7):448-453 
9. Randelli P, Landi S, Fanton F, Hoover GK, Morandi M (1999) Treatment of ipsilateral femoral neck and shaft fractures with the Russell-Taylor reconstructive nail. Orthopedics 22(7):673-676

10. Shetty MS, Kumar MA, Ireshanavar SS, Sudhakar D (2007) Ipsilateral hip and femoral shaft fractures treated with intramedullary nails. Int Orthop 31:77-81

11. Starr AJ, Bucholz RW (2001) Fractures of the shaft of the femur. In: Bucholz RW, Heckman JD (eds) Rockwood and Green's fractures in adults. Lippincott Williams \& Wilkins, Philadelphia, PA, pp 1683-1730

12. Watson JT, Moed BR (2002) Ipsilateral femoral neck and shaft fractures: complications and their treatment. Clin Orthop 399:78-86
13. Whittle AP, Wood GWII (2003) Fractures of lower extremity. In: Canale ST (ed) Campbell's operative orthopaedics. Mosby, St. Louis, MO, pp 2725-2872

14. Wolinsky PR, Johnson KD (1995) Ipsilateral femoral neck and shaft fractures. Clin Orthop Relat Res 318:81-90

15. Wu CC (2004) Ununited ipsilateral femoral neck and shaft fractures: treatment of 16 patients. Arch Orthop Trauma Surg 124(3): 173-178

16. Wu CC, Shih CH (1991) Ipsilateral femoral neck and shaft fractures. Acta Orthop Scand 62(4):346-351 